\title{
ERRATUM
}

Sawraj Singh · Gurmeet Kaur · Asit K. Chakraborti

Rakesh K. Jain · Uttam C. Banerjee

\section{Study of the experimental conditions for the lipase production by a newly isolated strain of Pseudomonas aeruginosa for the enantioselective hydrolysis of ( \pm )-methyl trans-3(4-methoxyphenyl) glycidate (MPGM)}

Published online: 1 March 2006

(C) Springer-Verlag 2006

\section{Bioprocess Biosyst Eng (2005)}

DOl: 10.1007/s00449-005-0039-7

Due to an unfortunate error some part of 16S rRNA (233-244) sequence have been incorrectly given. The correct sequence is printed below.

GGCACCCCTGGACTGATCTGACACTGAGGT GCGAAGCGTGGGGAGCAAACAGGATTAGATA CCCTGGTAGTCCACGCCGTAAACGATGTCGA CTAGCCGTTGGGATCCTTGAGATCTTAGTGGC GCAGCTAACGCGATAAGTCGACCGCCTGGGG
AGTACGGCCGCAAGGTTAAAACTCAAATGAAT TGACGGGGGCCCGCACAAGCGGTGGAGCATG TGGTTTAATTCGAAGCAACGCGAAGAACCTTA CCTGGCCTTGACATGCTGAGAACTTTCCAGAG ATGGATTGGTGCCTTCGGGGAACTCAGACACA GGTGCTGCATGGCTGTCGTCAGCTCGTGTCGT GAGATGTTGGGTTAAGTNCCGGTAACGAGCG CAACCCTTGTCCTTAGTTTACCAGCACCTCGGG GTGGGCACTCTAAGGAGACTGCCGGTGACCA $\mathrm{ACC}$

The online version of the original article can be found at http:// dx.doi.org/10.1007/s00449-005-0039-7

S. Singh · U. C. Banerjee $(\square)$

Biocatalysis Laboratory, Department of Pharmaceutical

Technology (Biotechnology), National Institute of Pharmaceutical Education and Research (NIPER), Sector 67, S. A. S. Nagar, 160062 Punjab, India

E-mail: ucbanerjee@niper.ac.in

Tel.: +91-172-2214682

G. Kaur · A. K. Chakraborti

Department of Medicinal Chemistry,

National Institute of Pharmaceutical Education and Research (NIPER), Sector 67, S. A. S. Nagar,

160062 Punjab, India

R. K. Jain

Institute of Microbial Technology (IMTECH), Sector 39-A, Chandigarh, 160036 India 